\title{
Retropharyngeal Space Hematoma in a Patient with Severe COVID-19 Illness
}

\section{BV Manjula*}

Senior Consultant and Head of the Department of ENT and Head and Neck Surgery, Bangalore Baptist Hospital, India

*Corresponding Author: BV Manjula, Senior Consultant and Head of the Department of ENT and Head and Neck Surgery, Bangalore Baptist Hospital, India.
Received: July 15, 2021

Published: August 28, 2021

(C) All rights are reserved by BV Manjula.

\begin{abstract}
Background: Deep neck spaces include prevertebral, danger and retropharyngeal spaces. Imaging cannot differentiate between these various fascial planes. A hematoma in these potential spaces due to any reason can compress the airway leading to stridor, dyspnea, neck pain and dysphagia. Most of these hematomas resolve within 2 weeks but few would require intervention depending on severity of symptoms.

Case Description: A 53 years old female patient was mechanically ventilated and tracheostomized for severe COVID-19 illness. She developed throat pain, swallowing difficulty and difficulty in neck movements, which were noticed after she was shifted to the ward. She was referred to the ENT department for endoscopic evaluation and imaging which suggested a large retropharyngeal collection. She underwent intraoral aspiration and drainage of hematoma after which her symptoms resolved.

Conclusion: Individualized approach is needed for these rare conditions and sometimes a simple procedure may be sufficient to address a major, possibly life threatening condition.
\end{abstract}

Keywords: Retropharyngeal Hematoma; Prevertebral Space; Dysphagia; Airway Obstruction; Anticoagulants

\section{Introduction}

Retropharyngeal hematomas are rare conditions and airway obstruction should be anticipated in all those patients regardless of age, severity of trauma, or lack of symptoms especially in those with cervical spine injuries because of the close relation of the retropharyngeal space to the airway [1]. Any swelling in this region will cause the posterior pharyngeal wall to bulge anteriorly into the laryngeal airway leading to obstruction. Most of these hematomas resolve with time but few would require intervention depending on the site, size, duration and severity of symptoms.

Here we present a unique case of retropharyngeal/prevertebral space hematoma in a patient with severe COVID-19 illness prob- ably secondary to intubation injury with prolonged anticoagulant use and prone ventilation which delayed the diagnosis and treatment.

\section{Case Report}

A 53 years old obese female patient with short neck and comorbidities (diabetes and hypertension) presented to the emergency unit with cough, shortness of breath for 1 week. She was desaturating and collapsed even with $100 \%$ oxygen. Therefore, urgent intubation was done and ventilated. She was diagnosed to be COVID-19 positive and was shifted to COVID-19 ICU. Staying prone-positioned, she developed venous stasis, macroglossia for which she was tracheostomized. She was weaned off ventilator after 8 days of 
admission. Since she was hemodynamically stable, she was shifted to COVID-19 isolation ward a week later. The following week, she was shifted to the regular ward after having improved.

In spite of 2 weeks of ward stay and clinically better pulmonary status, she was still in need of oxygen and desaturated on being recumbent. She was also on Nasogastric (NG) feeding. She had persistent sore throat, dysphagia and difficulty in neck movement. She was referred to the ENT department for evaluation. On clinical examination and flexible endoscopy, pooling of saliva and diffuse posterior pharyngeal wall swelling were noticed. Larynx and hypopharynx could not be visualized. Therefore, contrast enhanced computed tomography (CECT) neck and chest was done (Figure 1) which showed prevertebral collection from second cervical (C2) to fourth thoracic vertebrae (T4). A spinal surgeon was consulted as probably these collections could be secondary tuberculosis or vertebral injury but all other differential diagnoses were ruled out.

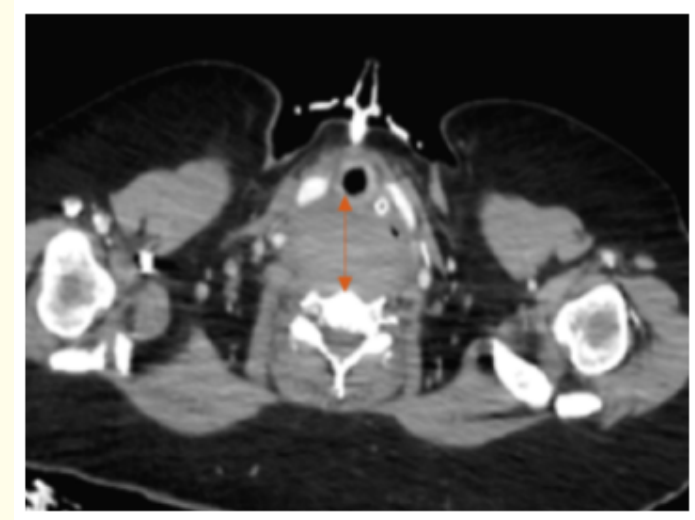

Figure 1: CECT scan suggestive of a large prevertebral collection.

MRI neck and chest (Figure 2) was also done which showed a similar picture and confirmed the large collection as hematoma causing compression of adjacent carotid and jugular vessels laterally, mass effect with compression of the oropharyngeal and laryngeal airway, anterior displacement and indentation of the oesophagus. In view of hematoma, she was planned for intra oral/external drainage under general anaesthesia. Per operatively, a linear tear with old clots was noticed in the right side of posterior pharyngeal wall probably secondary to intubation injury through which an Yankauer suction tube was inserted and large clots were drained. Later, rigid endoscope was introduced through the rent. Inferior and lateral clots were completely suctioned out under vision. Romsons drain was placed through the mucosal breech into the retropharyngeal space. Mucosa was sutured with vicryl 3.0. The drain tube was brought out through the right nostril and secured. Postoperatively, she was shifted to the ICU for observation. On the fourth postoperative day, after her symptoms subsided, flexible scopy was done and drain was removed. Postoperatively, CECT scan (Figure 3) was done to confirm complete drainage and rule out residual or recurrent collection. It showed complete resolution of hematoma. Swallowing therapy was initiated, NG tube was removed, tracheostomy was decannulated and the patient was discharged.

\section{Discussion}

Aetiology of retropharyngeal hematoma includes infection, cervical spine/great vessel trauma, violent head movements, iat-

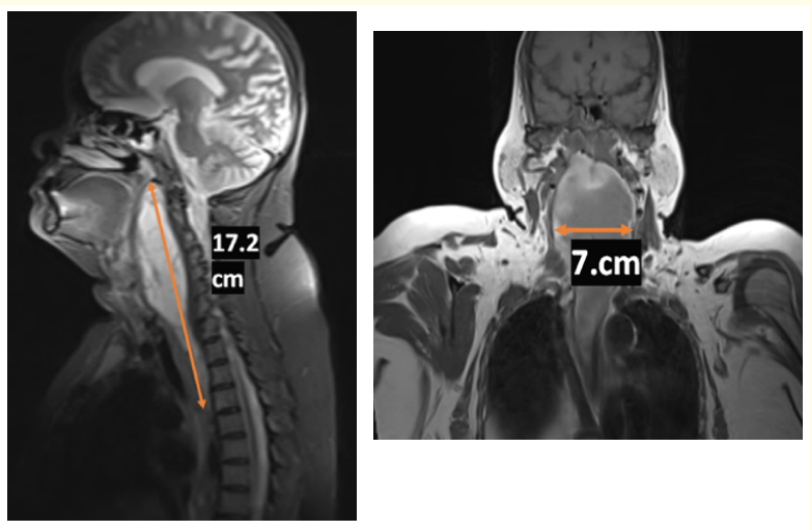

Figure 2a and 2b: MRI neck and chest suggestive of a large prevertebral collection measuring $17.2 \mathrm{~cm}$ (CC) x $3.5 \mathrm{~cm}$ (AP) $\mathrm{x} 7 \mathrm{~cm}(\mathrm{Tr})$ extending craniocaudally from the upper border of $\mathrm{C} 2$ inferiorly across the thoracic inlet to the posterior mediastinum, with a thin linear extension upto the lower border of T4. Mass effect with compression of the oropharyngeal and laryngeal airway, anterior displacement and indentation on the esophagus. Laterally, there is compression and displacement of the carotid vessels and jugular veins. 


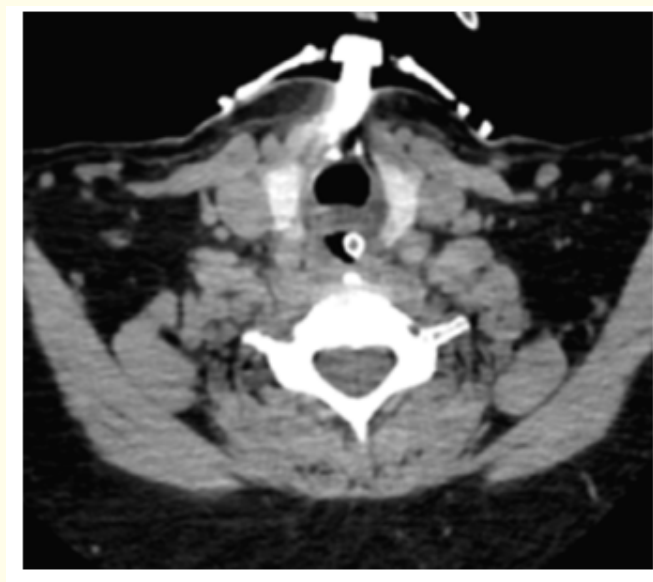

Figure 3: Post-operative CECT scan showed tracheostomy tube and NG in situ with no residual collection.

rogenic injury, parathyroid adenoma hemorrhage, and foreign body ingestion and migration [1]. Etiologically, prevertebral space hematoma is seen exclusively in fractures of the odontoid process or vertebral bodies. In case of hyperextension injuries, it has been established that disruption of anterior ligaments and probable fractures of anterior bony elements at C1 to C4 can lead to massive hematomas [2]. The relation between large retropharyngeal hematomas and disruptive hyperextension sprain is also well known.

The potential deep neck spaces are separated by various fascial layers from the vertebral bodies and the pharyngeal musculature. These spaces are difficult to distinguish radiologically [3]. The prevertebral space's posterior border is formed by the vertebral bodies and their ligaments. Although these potential spaces are not usually separate, blood may enter them if their integrity is violated by trauma [4]. Retropharyngeal/prevertebral hematomas, either due to trauma or anticoagulant use, are life threatening due to airway compromise.

Due to its rarity of occurrence, opinions are divided and they have no particular management protocol. A customized treatment plan is required for each patient based on the symptoms, size and extent of the collection. Management should start with securing and maintaining the patient's airway. Diagnosis depends on clini- cal evaluation and radiology findings whereas treatment depends on severity of symptoms and size of collections. Observation is advocated in all mild cases but few believe early tracheostomy is good in patients with warning signs [5]. Some also suggest a 2 to 4 week observation prior to surgical intervention for drainage of expansile hematomas [2,6-8]. Large hematomas that fail to resolve should undergo drainage. Two methods of drainage have been described; transoral aspiration and external drainage. Transoral aspiration seems better suited to smaller hematomas and may reduce recovery time but does carry a risk of bacterial contamination of the retropharyngeal space. The external lateral cervical approach appears better suited for larger hematomas and prevents pharyngeal contamination of the retropharyngeal space [7].

The possible aetiology in this patient could be intubation injury of the posterior pharyngeal wall secondary to the emergent nature of COVID-19 illness with associated patient factors like short neck. There also could have been probable damage of the anterior vertebral ligaments due to hyperextension while difficult intubation in emergency leading to violation of the boundaries of potential spaces and thereby progressing to massive collection. Additionally, prone positioning advocated for COVID-19 along with anticoagulant therapy could have aggravated the retropharyngeal collection.

Securing the airway is the primary step for any large retropharyngeal collections which lead to life threatening airway compromise. Due to the severity of her COVID-19 illness, she was mechanically ventilated and later tracheostomized which had her airway secured. She was also on NG feeding which shadowed the presence of laryngeal airway compromise. Hence, there was a delay in diagnosis till the patient recovered from COVID-19 and complained of severe pain and dysphagia following which she was evaluated and managed surgically.

\section{Conclusion}

Retropharyngeal hematoma is a potentially fatal situation which warrants early suspicion, diagnosis and prompt management even though injuries pertaining to cervical vertebrae or inner pharyngeal mucosal layer may be trivial in nature. These rare cases always present with the dilemma of an intraoral or external cervical approach for drainage. Though external lateral cervical approach is recommended for large collections, we could avoid it in this patient because of the linear tear noticed in the posterior pharyngeal wall 
through which endoscopic guided complete evacuation of the hematoma was possible. Difficult traumatic intubation or inappropriate prone positioning of COVID-19 patients with tracheostomy can inadvertently lead to iatrogenic induced retropharyngeal hematomas in those with anticoagulant therapy as in this case.

\section{Declaration of Patient Consent}

The authors certify that they have obtained appropriate patient consent form.

\section{Financial Support and Sponsorship}

Nil.

\section{Conflicts of Interest}

There are no conflicts of interest.

\section{Bibliography}

1. Kuhn JE and Graziano GP. "Airway compromise as a result of retropharyngeal hematoma following cervical spine injury". Journal of Spinal Disorders 4.3 (1991): 264-269.

2. Penning L. "Prevertebral hematoma in cervical spine injury: incidence and etiologic significance". AJR: American Journal of Roentgenology 136.3 (1981): 553-561.

3. Shaw CB., et al. "Traumatic Retropharyngeal Hematoma: A Case Report". Otolaryngology- Head and Neck Surgery 113.4 (1995): 485-488.

4. Myssiorek D and Shalmi C. "Traumatic Retropharyngeal Hematoma". Archives of Otolaryngology - Head and Neck Surgery 115.9 (1989): 1130-1132.

5. Cohen AF and Warman SP. "Upper airway obstruction secondary to warfarin-induced sublingual hematoma". Archives of Otolaryngology - Head and Neck Surgery 115.6 (1989): 718720.

6. Ophir D and Bartal N. "Retropharyngeal hematoma following fish-bone ingestion". Ear, Nose and Throat Journal 67.7 (1988): 528-530.

7. Daniello NJ and Goldstein SI. "Retropharyngeal hematoma secondary to minor blunt head and neck trauma". Ear, Nose and Throat Journal 73.1 (1994): 41-43.
8. O'Neill JV., et al. "Retropharyngeal hematoma secondary to minor blunt trauma in the elderly patient". Journal of Otolaryngology 6.1 (1977): 43-46.

\section{Volume 3 Issue 9 September 2021}

(C) All rights are reserved by BV Manjula. 